\title{
Consumo abusivo de alcohol en estudiantes adolescentes
}

\author{
Abusive of alcohol in adolescent students \\ María Auxiliadora Arrom Suhurt $\mathbb{B}^{1}$, María del Pilar Fresco $\mathbb{D}^{2}$, Carmen Marina Arrom Suhurt $\mathbb{1}^{1}$, Mónica Ruoti(D) \\ Marcos Hernán Capurro $\mathbb{D}^{3}$, Cristina Arrom Suhurt $\mathbb{1}^{4}$, Manuel Ángel Fresco Ortiz $\mathbb{D}^{3}$ \\ ${ }^{1}$ Centro para el Desarrollo de la Investigación Científica, Asunción, Paraguay. \\ ${ }^{2}$ Univerisdad Católica Nuestra Señora de la Asunción, Asunción, Paraguay \\ ${ }^{3}$ Centro Nacional de Prevención, Tratamiento y Rehabilitación de Adicciones, Asunción, Paraguay \\ ${ }^{4}$ Instituto de Investigaciones en Ciencias de la Salud, San Lorenzo, Paraguay
}

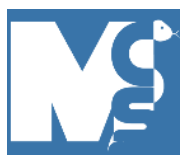

Recibido: $11 / 06 / 2020$

Revisado: 19/08/2020

Aceptado:08/12/2020

\section{Autor correspondiente}

María Auxiliadora Arrom Suhurt Centro para el Desarrollo de la Investigación Científica

maryarrom@yahoo.com.ar

\section{Conflictos de interés}

Los autores declaran no poseer conflictos de interés.

\section{Fuente de financiación}

Los autores no recibieron apoyo financiero para la investigación, autoría y/o publicación de este artículo.

Este es un artículo publicado bajo una licencia de Creative Commons Reconocimiento 4.0 Internacional.

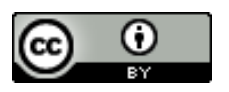

\section{RESUMEN}

Introducción: el consumo nocivo de alcohol en adolescentes preocupa cada vez más en muchos países, pues reduce el autocontrol y aumenta los comportamientos de riesgo. Objetivo: se propuso analizar el consumo abusivo de alcohol en 463 estudiantes de secundaria. Metodología: estudio transversal descriptivo y retrospectivo, en adolescentes de 56 colegios del Departamento de Alto Paraná. Muestreo probabilístico, trietápico, cuestionario auto-administrado, estructurado y anónimo, previa autorización. Para nivel de consumo se utilizaron como parámetro, cantidades de consumo abusivo de alcohol de la Encuesta Nacional de Hábitos Tóxicos del Paraguay. Información cargada en base de datos en Excel, analizada con Stata. Resultados: Del total de 661 de la muestra, 70,0 \% (463) consumió alguna vez en su vida, $27 \%$ (182) no. De este total (100\%/463) 75,6 \% (350) consumió con 15 años (y menos) y desde los 16 años, el $19 \%$ (88). En el último año, 44,7 \% (207) consumió cerveza en forma abusiva; 34,3 \% (159) consumió menos y 17,9 \% (83) no consumió. También 35,0 \% (162) consumió vino en forma abusiva, 28,7 \% (133) consumió menos y 32,4 \% (150) no consumió. En los últimos 30 días, consumieron cerveza en forma abusiva 42,1 \% (195), mientras $24,4 \%$ (113) consumió menos y $28,9 \%$ (134) no consumió. También 29,2 \% (135) consumió vino en forma abusiva, mientras $21,4 \%$ (99) menos y $43,8 \%$ (203) no consumió. Conclusión: consumo abusivo de alcohol que se observa a temprana edad en la población de estudio y consumo femenino que prácticamente iguala al consumo de varones.

Palabras clave: alcohol; abuso; adolescentes; estudiantes

\section{ABSTRACT}

Introduction: the harmful consumption of alcohol in adolescents is more and more worrisome in many countries, since it reduces self-control and increases the risk behaviors. Objective: it was proposed to analyze the abusive consumption of alcohol in 463 high school students. Methodology: descriptive and retrospective cross-sectional study in adolescents from 56 schools in the Department of Alto Paraná. Probabilistic, threestage sampling, self-administered, structured and anonymous questionnaire, with prior authorization. For consumption level, alcohol abuse amounts from the National Survey of Toxic Habits of Paraguay were used as a parameter. Information uploaded to Excel database, analyzed with Stata. Results: Of the total of 661 in the sample, $70,0 \%$ (463) consumed at some time in their lives, $27 \%(182)$ did not. Of this total $(100 \% / 463)$ $75,6 \%$ (350) consumed with 15 years (and less) and from 16 years, 19\% (88). In the last year, 44,7\% (207) consumed beer in an abusive way; $34,3 \%$ (159) consumed less and $17,9 \%(83)$ did not consume. Also $35,0 \%$ (162) consumed wine in an abusive way, $28,7 \%$ (133) consumed less and 32,4\% (150) did not consume. In the last 30 days, $42,1 \%$ (195) consumed beer ?busively, while $24,4 \%$ (113) consumed less and $28,9 \%$ (134) did not consume. Also $29,2 \%$ (135) consumed wine in an abusive way, while $21,4 \%$ (99) less and $43,8 \%$ (203) did not consume. Conclusion: abusive alcohol consumption observed at an early age in the study population and female consumption that practically equals the consumption of men.

Keywords: alcohol; abuse; adolescents; students. 


\section{INTRODUCCIÓN}

Datos de la Organización Mundial de la Salud indican que cada año mueren 3,3 millones de personas en el mundo debido al consumo nocivo de alcohol, lo que representa 5,9 \% de todos los fallecimientos. Es también un factor causal de más de 200 enfermedades y trastornos (1).

Aproximadamente uno de cada seis habitantes del mundo es un adolescente, lo que representa que 1.200 millones de personas tienen entre 10 y 19 años (2). Los jóvenes en su mayoría gozan de buena salud, pero la mortalidad y morbilidad entre los mismos sigue siendo elevada y el consumo de alcohol puede afectar su salud actual, así como la de sus futuros hijos (2).

Maristela Monteiro, asesora en abuso de sustancias y alcohol de la OMS señaló que el alcohol es el principal factor de riesgo de muerte para los adolescentes de 15 a 19 años de edad (3). Específicamente el 7,2 \% de la mortalidad precoz en el mundo se produjo como consecuencia del consumo de alcohol en personas de 69 años y menores. En efecto los más jóvenes fueron afectados de manera excesiva por el alcohol con respecto a los adultos.

De todas las muertes atribuibles al alcohol en el mundo, el $28 \%$ fueron como consecuencia de lesiones, como las causadas por accidentes de tráfico, autolesiones y violencia interpersonal; el $21 \%$ por trastornos digestivos; el $19 \%$ debido a enfermedades cardiovasculares, y el resto a enfermedades infecciosas, cánceres, trastornos mentales y otras afecciones (4).

En el primer informe sobre alcohol especializado en la región, elaborado por el organismo, las cifras dan cuenta que en América Latina y el Caribe el consumo por persona es de una media de 8,4 litros de alcohol puro por año, 2,2 litros más que el promedio mundial. Los países que más ingieren en la región, con un consumo anual per cápita son Chile 9,6 litros; Argentina 9,3 litros; Venezuela 8,9 litros y Paraguay en cuarto lugar con 8,8 litros de alcohol per cápita (3).

Un estudio realizado por Romero en Azogues, Ecuador, encontró que la prevalencia de consumo de alcohol en estudiantes del colegio Ezequiel Cárdenas Espinoza, en el periodo lectivo 2011; 2012, fue de 23,3 \%. Este dato se ubica muy por encima de los trabajos realizados por el CONSEP (Consejo Nacional de Sustancias Estupefacientes y Psicotrópicas) de Ecuador (5). Y el estudio de Pardo Jaime con 1193 estudiantes de Bogotá, Colombia, que cursaban entre el sexto grado y el onceavo de secundaria, de colegios públicos y privados, mostró que los motivos sociales para sobresalir son los factores predisponentes para consumir alcohol, así como para manejar problemas (6).

Con respecto a Paraguay respecto al consumo de alcohol en estudiantes, investigaciones mostraron que más de la mitad de los mismos consumió alcohol en el último año según una investigación elaborada por la Comisión Interamericana para el Control del Abuso de Drogas (CICAD) de la OEA, que reveló el alto consumo en jóvenes de entre 13 y 17 años (7).

Y en referencia a lo señalado más arriba, datos proporcionados por el Ministerio de Salud Pública de Paraguay mostraron resultados de la Encuesta Global de Salud Escolar aplicada a 49 escuelas y colegios públicos y privados de 12 departamentos del país y Asunción (8). Los resultados son considerados alarmantes y preocupantes, ya que arrojaron una alta prevalencia del consumo de alcohol de 46,7 \% en escolares que admitieron haber consumido alguna vez bebidas alcohólicas y lo hicieron por primera vez antes de los 14 años. En los últimos 30 días $35 \%$ de ellos ingirió alcohol, con leve predominio de varones, mientras 83 \% inició el consumo en octavo grado y 53,3 $\%$ que consumió bebidas alcohólicas, estuvieron ebrios una o más veces y manifestaron haber tenido contacto sexual en estado de ebriedad (8).

Con base en las consecuencias que acarrea el consumo abusivo de alcohol en adolescentes, se propuso analizar en un contexto educativo de 56 colegios del Departamento de Alto Paraná, las características del mismo, proponiendo como objetivo analizar el consumo abusivo de 463 estudiantes de secundaria que (de un total de 661 consultados y consultadas) respondieron haber consumido alguna vez.

\section{METODOLOGÍA}

Estudio descriptivo y retrospectivo. Se tomaron datos con fuente secundaria de un estudio de adolescentes en 56 colegios de las ciudades Minga Guazú, Presidente Franco, Hernandarias y Ciudad del Este del departamento de Alto Paraná en Paraguay, analizando las variables de consumo de alcohol.

Un cuestionario auto; administrado, estructurado y anónimo, previa autorización de padres fue presentado a los Estudiantes. Información cargada en base de datos en Excel, analizada con STATA. Para determinar el tamaño de la muestra en el estudio de base se utilizó el paquete Epidat (Organización Panamericana de la Salud, Junta de Salud de Galicia y Universidad CES de Colombia) con un nivel de confianza de $95 \%$ y una proporción esperada de 0,50. 
La muestra quedó en 600 casos, a los que se agregó un excedente en previsión de invalidaciones y reemplazos. Para el procesamiento estadístico la muestra se cerró en 661 casos. El diseño de muestreo fue probabilístico trietápico (9), cuyo desarrollo fue el siguiente: En la primera etapa fueron seleccionados los colegios en forma sistemática; en la segunda etapa fueron elegidos los cursos dentro de cada colegio en forma sistemática; en la tercera etapa, fueron seleccionados los alumnos estratificados por edad y sexo en cada curso, en base a la lista proporcionada por el Ministerio de Educación y la Supervisión de cada ciudad.

Se completó un cuestionario auto; administrado, estructurado, anónimo previa autorización. Se solicitó a estudiantes que participaran de forma voluntaria, proporcionándoles información necesaria sobre la administración confidencial de los resultados y los objetivos de la investigación. Las variables socio demográficas fueron sexo, grupo etario, personas con quienes vive.

Para conocer el nivel de consumo abusivo de alcohol se utilizaron como parámetro las cantidades de abuso establecidas en la Encuesta Nacional de Salud Mental y Hábitos tóxicos en Paraguay (10). Se tomó como referencia la ingestión de $100 \mathrm{cc}$ de alcohol absoluto en una oportunidad, aclarando los volúmenes diferentes que esto podía significar de acuerdo al tipo de bebida en cuestión (vino, cerveza, destilado). Estos $100 \mathrm{cc}$ de alcohol absoluto se expresaron en las unidades populares, cómo son pedidos y servidos, especialmente en el caso de las bebidas destiladas donde no se parte de un envase que fija la cantidad de una forma exacta. Se determinó un límite de 23; 26 cc de alcohol absoluto por cada "raya" de whisky o caña servida en bares de diferentes sectores sociales. Con respecto a la fijación de los $100 \mathrm{cc}$ de alcohol absoluto (más de un cuarto de bebida destilada, de un litro de vino o de dos litros de cerveza) como cantidad a partir de la cual puede intentarse una delimitación operativa entre el uso y el abuso, se sostiene que la cantidad de alcohol puede medirse en términos de porcentaje de calorías aportadas por el alcohol a la dieta diaria del individuo, pudiendo fijarse arbitrariamente en $20 \%$ el límite entre ingestión moderada y excesiva (10). El hombre que consume en la práctica 3.000 calorías derivaría del alcohol no más de 600 calorías diarias si permanece como bebedor moderado. Como cada gramo de alcohol oxidado libera en el organismo 7,1 calorías, el total de alcohol absoluto ingerido no debería exceder en este caso los 100 cc u 80 gramos, lo cual equivale aproximadamente a un litro de vino (con $10 \%$ de alcohol), o $1 / 4$ litro de bebidas destiladas (con $40 \%$ de alcohol) o dos litros de cerveza (con $5 \%$ de alcohol) (10). La información fue cargada en una planilla electrónica (Excel Microsoft) y analizada con programa estadístico informático STATA.

Control de calidad de los datos: como método el instrumento de campo fue sometido a prueba con población de 50 estudiantes de Alto Paraná, quienes fueron excluidos en la aplicación definitiva. Luego se elaboró la versión final del instrumento. A medida que se completaban los cuestionarios se realizaba una crítica a fin de detectar inconsistencias, luego fueron preparados para su digitalización.

Consideraciones Éticas: en los meses previos a la implementación, se obtuvo una autorización del Ministerio de Educación y Ciencias (MEC), que declaró de interés el desarrollo del Estudio. Posteriormente desde cada Institución (56 colegios) se envió una Nota a padres y madres para solicitar autorización a los/as mismos/as. La participación de los/las adolescentes fue voluntaria, proporcionándoles información suficiente y clara sobre la utilización de los datos y fines de la investigación. En todos los casos se guardó escrupulosamente la confiabilidad de las informaciones obtenidas. Los autores declaran no tener conflictos de interés comercial. El estudio no cuenta con financiación más que el Incentivo a los Investigadores PRONII CONACYT.

\section{RESULTADOS}

En un total de 661 estudiantes del nivel medio, respondieron haber consumido bebidas alcohólicas alguna vez en la vida un $70 \%$ (463) y un $27,5 \%$ (182) no lo ingirió, el resto no contesta. De 463 estudiantes que consumieron alguna vez en su vida alcohol, 244 $(52,7 \%)$ eran del sexo masculino y $219(47,3 \%)$ del sexo femenino, con edades comprendidas mayormente entre 16 a 17 años 44,4 \% (205). Una mayoría $70,8 \%$ (328) vivía con el padre y la madre (Tabla 1). 
TABLA 1. DATOS SOCIODEMOGRÁFICOS DE LOS QUE CONSUMIERON BEBIBDAS ALCOHÓLICAS ALGUNA VEZ (N=661)

\begin{tabular}{ccc}
\hline Sexo & Frecuencia & Porcentaje \\
\hline Hombre & 244 & 52,7 \\
Mujer & 219 & 47,3 \\
Edad & & \\
$12-13$ años & 35 & 7,6 \\
$14-15$ años & 121 & 26,1 \\
$16-17$ años & 205 & 44,4 \\
Más de 17 años & 100 & 21,5 \\
No contesta & 2 & 0,4 \\
Personas con quienes viven & & \\
Padre y Madre & 328 & 70,8 \\
Padre & 12 & 2,6 \\
Madre & 71 & 15,3 \\
Parientes & 30 & 6,5 \\
Otros & 22 & 4,8 \\
\hline
\end{tabular}

El inicio en el consumo de alcohol se dio mayormente en el rango de 13 a 15 años con 52,9\% (245) y en niños/as menores de 8 años el 0,9\% (4) (Tabla 2). En el último año un $44,7 \%$ (207) de estudiantes consumieron 2 botellas o 6 latitas de cerveza; el 35\% (162) dijo haber consumido 1 botella o más de vino; el 14,7\% (68) consumió 4 rayas de caña o whisky (Tabla 3). En cuanto a los últimos 30 días un 42,1\% (195) de estudiantes consumió 2 botellas o 6 latitas de cerveza; el 29,2\% (135) dijo haber consumido 1 botella o más de vino; un $13,2 \%(61)$ consumió 4 rayas de caña o whisky (Tabla 4)

TABLA 2. EDAD QUE TENÍA LA PRIMERA VEZ QUE CONSUMIÓ ALCOHOL (N=463).

\begin{tabular}{ccc}
\hline $\begin{array}{c}\text { Edad que tenía la primera vez que } \\
\text { consumió alcohol }\end{array}$ & Frecuencia & Porcentaje \\
\hline Menos de 8 años & 4 & 0,9 \\
De 8 a 12 años & 101 & 21,8 \\
De 13 a 15 años & 245 & 52,9 \\
De 16 a 18 años & 85 & 18,4 \\
Más de 18 años & 3 & 0,6 \\
No responde & 25 & 5,4 \\
\hline
\end{tabular}


TABLA 3. CONSUMO DE BEBIDAS ALCOHÓLICAS EN EL ÚLTIMO AÑO (N=463)

\begin{tabular}{|c|c|c|c|c|c|c|c|c|}
\hline \multirow{2}{*}{ Consumo de bebidas alcohólicas en el último año } & \multicolumn{2}{|c|}{ Sí } & \multicolumn{2}{|c|}{ No } & \multicolumn{2}{|c|}{ Menos } & \multicolumn{2}{|c|}{ No Contesta } \\
\hline & № & $\%$ & № & $\%$ & № & $\%$ & № & $\%$ \\
\hline *2 Botellas o 6 latitas de cerveza con $5 \%$ de alcohol & 207 & 44,7 & 83 & 17,9 & 159 & 34,3 & 14 & 3,0 \\
\hline *1 botella o más de vino con $10 \%$ de alcohol & 162 & 35,0 & 150 & 32,4 & 133 & 28,7 & 18 & 3,9 \\
\hline$* 4$ rayas de caña o whisky con $40 \%$ de alcohol & 68 & 14,7 & 289 & 62,4 & 88 & 19,0 & 18 & 3,9 \\
\hline
\end{tabular}

TABLA 4. CONSUMO DE BEBIDAS ALCOHÓLICAS EN LOS ÚLTIMOS 30 DÍAS (N=463)

\begin{tabular}{|c|c|c|c|c|c|c|c|c|}
\hline \multirow{2}{*}{$\begin{array}{l}\text { Consumo de bebidas alcohólicas en los últimos } 30 \\
\text { días }\end{array}$} & \multicolumn{2}{|c|}{ Sí } & \multicolumn{2}{|c|}{ No } & \multicolumn{2}{|c|}{ Menos } & \multicolumn{2}{|c|}{$\begin{array}{l}\text { No } \\
\text { Contesta }\end{array}$} \\
\hline & № & $\%$ & № & $\%$ & № & $\%$ & № & $\%$ \\
\hline *2 Botellas o 6 latitas de cerveza con $5 \%$ de alcohol & 195 & 42,1 & 134 & 28,9 & 113 & 24,4 & 21 & 4,5 \\
\hline$* 1$ botella o más de vino con $10 \%$ de alcohol & 135 & 29,2 & 203 & 43,8 & 99 & 21,4 & 26 & 5,6 \\
\hline$* 4$ rayas de caña o whisky con $40 \%$ de alcohol & 61 & 13,2 & 302 & 65,2 & 72 & 15,6 & 28 & 6,0 \\
\hline
\end{tabular}

\section{DISCUSIÓN}

Quedó constatado en el presente estudio un alto porcentaje de personas que consumían 2 botellas o 6 latitas de cerveza, 1 botella o más de vino y 4 rayas de caña o whisky, lo que equivalen a $100 \mathrm{cc}$ u 80 gramos de alcohol absoluto considerado consumo abusivo. Estos resultados son similares a los hallados en el trabajo llevado a cabo por Olivares que constató abuso de alcohol en los adolescentes, $16,2 \%$ de ellos se había embriagado en el último año y $8,9 \%$ en los últimos treinta días (11). No obstante, en un estudio realizado en Río Grande Do Sul, Brasil, con 710 estudiantes de primaria y edades comprendidas entre 10 y 13 años se encontraron porcentajes mucho más elevados que el presente estudio (62,8 \%), (2011). El consumo en el último mes y la ocurrencia de embriaguez fue mayor en hombres que en mujeres, aunque esta diferencia no fue estadísticamente significativa (12).

Otro hallazgo fue en el estudio de Morello realizado con 3172 estudiantes de las ciudades de Buenos Aires, Córdoba y Tucumán, Argentina, cuya edad promedio fue de 12,8 años, mostró que en los 30 días previos a la encuesta el $32 \%$ había consumido alcohol y $17 \%$ tuvo un episodio excesivo de consumo (13). Concordante además con otra fuente como la Quinta Encuesta Nacional a Estudiantes de Enseñanza Media, 230 adolescentes de entre 13 a 17 años, realizada en Argentina, los resultados concluyeron que el alcohol fue la sustancia psicoactiva de mayor consumo intensivo con $63,4 \%$ y presentaba la edad de inicio de consumo de alcohol más baja: 13 años (14).

Los resultados del estudio dejan además en evidencia que el consumo femenino prácticamente se iguala al consumo masculino. Las mujeres beben cada vez más en muchos países, en la misma proporción que los hombres. Sin embargo, esa igualdad conlleva más desigualdad entre los sexos en lo que respecta a las consecuencias para la salud. En las Américas las mujeres presentan una prevalencia mucho mayor del trastorno relacionado con consumo de alcohol. Por otro lado, sobre el consumo de alcohol en las mujeres, recae un estigma mayor que el de los hombres y tienen más barreras al momento de acudir a los servicios de ayuda (15).

Cada vez más mujeres tienen comportamientos relacionados con el consumo abusivo de alcohol, lo que 
presupone un doble riesgo. Primero porque las mujeres desarrollan un alcoholismo mucho más rápido que los varones. Segundo porque la mujer tiene la función materna, entonces el embarazo y el consumo son un doble riesgo, ya que el bebé en crecimiento recibe el alcohol y también sufre los efectos en el vientre materno (15).

El inicio de consumo de alcohol se dio a temprana edad en el grupo de estudio, lo que coincide con estudios nacionales donde se identificó que en los últimos años en Paraguay ha disminuido la edad de inicio del consumo, situándose el promedio en 13 años (7). Al mismo tiempo el llevado a cabo por Fernández Raone en la ciudad de La Plata Argentina, mostró que la edad de inicio de la mayoría de los consultantes (63\%) había sido entre los 12 y 15 años, momento donde nacen las primeras expresiones del despertar de la pubertad (16). De acuerdo con el estudio de Rueda Jaimes llevado a cabo en Bucaramanga, Colombia, en diferentes instituciones educativas, con 2.931 estudiantes de entre 10 y 22 años y una media de 14,4 años, los resultados mostraron que 51,1 \% eran mujeres y la edad de inicio de consumo fue de 12,9 años (17). También cabe comparar con el estudio de Olivares en 2.890 adolescentes de 10 centros educativos de secundaria en la ciudad de Madrid, España, que mostró una edad de inicio de consumo de alcohol a los 12, 06 años (11); así como con el estudio de Fernández Díaz en 227 adolescentes residentes de Consejo Popular Cayo Hueso de Centro Habana, Cuba, que reflejó inicio de consumo de alcohol en menores de 16 años (18).

Las afirmaciones de las evidencias anteriores sugieren que el abordaje estatal para frenar este consumo abusivo de alcohol por parte de los jóvenes, podría darse a través de un servicio de atención especializado en el tema. Otras medidas que contribuyan con la reducción del consumo serían: la suba de impuestos, límite de horarios y días de venta en establecimientos, restricción a la publicidad y promoción, límite de edad para compra y consumo, y realizar control de alcoholemia aleatorio como medida de prevención (4).

Finalmente atendiendo las recomendaciones del informe sobre la situación mundial del alcohol y la salud 2018 de la OMS en sus capítulos 1, 2, 5 y 6 que plantea el abordaje sobre consumo nocivo de alcohol, es importante resaltar que se precisa la participación de gobierno, sociedad e instituciones privadas que trabajen en forma coordinada en pos de políticas públicas que permitan un seguimiento eficaz de los Objetivos de la Agenda 2030 para el Desarrollo Sostenible (4).
Una limitación del presente estudio es que en su línea temporal es retrospectivo, hecho que constituye un obstáculo respecto a sus resultados que ya no responden a la realidad actual si se realizara el mismo con la misma población estudiantil en corte trasversal

\section{REFERENCIAS}

1. Organización Mundial de la Salud. Alcohol. Datos y cifras [Internet]. 2018 [citado 14 de enero de 2021]. Disponible en: sheets/detail/alcohol https://www.who.int/es/news-room/fact-

2. Organización Mundial de la Salud. Adolescentes: riesgos para la salud y soluciones [Internet]. 2018 [citado 14 de enero de 2021]. Disponible en: https://www.who.int/es/news-room/fact-

sheets/detail/adolescents-health-risks-and-solutions

3. Los países que más beben en América Latina: la dramática radiografía del consumo de alcohol en la región [Internet]. BBC News Mundo. 2015 [citado 14 de enero de 2021]. Disponible https://www.bbc.com/mundo/noticias/2015/07/150723 consumo alcohol latinoamerica muertes paises im

4. Organización Panamericana de la Salud. Informe sobre la situación mundial del alcohol y la salud 2018. 2019.

5. Romero M de LR, Ruiz AL. Abuso de alcohol e intervención mediante habilidades sociales en estudiantes del colegio "Ezequiel Cárdenas Espinoza" Azogues-Ecuador". Rev Fac Cienc Médicas Univ Cuenca. 2015;33(3):57-64. URL

6. Jaime LP, Ortega LM, Bernal C, Estupiñan DCM, Pisco JCR, Gómez MS. Motivos asociados al Consumo de Alcohol en estudiantes de la ciudad de Bogotá. Rev Psicol GEPU. 2016;7(2):1. URL

7. Observatorio Paraguayo de Drogas. Informe Nacional. Situación de drogas en Paraguay 2017. Secretaría Nacional Antidrogas [Internet]. 2018 [citado 14 de enero de 2021]. Disponible en: https://www.issup.net/files/201906/Informe\%20Nacional\%20Situaci\%C3\%B3n\%20de\%20Dr ogas\%20en\%20Paraguay\%202017.pdf

8. Ministerio de Salud Pública y Bienestar Social. Encuesta Global de Salud Escolar (GSHS) [Internet]. 2017 [citado 14 de enero de 2021]. Disponible en: https://www.mspbs.gov.py/dependencias/portal/adjunto/ e1e76b-EncuestaGlobalGSHSvistaporpagina.pdf

9. Hernández Sampieri R, Fernández Collado C, Baptista Lucio P. Metodología de la investigación. México: McGraw-Hill; 2007.

10. Miguez H, Pecci M, Carrizosa A, Gauto C. Estudio sobre salud mental y hábitos tóxicos en Paraguay. Asunción: Litocolor; 1992.

11. Olivares JU, Baena BC, Ursúa MP, Falcón CM. Estructura familiar y consumo de alcohol en adolescentes. Health Addict Salud Drog. 2018;18(1):107-18. URL.

12. Argimon II de L, Campana A, Estefenon S, Terroso LB, Lopes RMF, Terroso LB. Consumo de alcohol en niños y adolescentes de un municipio en el sur de brasil. Rev Argent Clínica Psicológica. 2016;XXV(3):267-74. URL.

13. Morello P, Pérez A, Peña L, Braun SN, Kollath-Cattano C, Thrasher JF, et al. Risk factors associated with tobacco, alcohol and drug use among adolescents attending secondary school in three cities from Argentina. Arch Argent Pediatr. 2017;115(2):155-8. Https://www.doi.org/10.5546/aap.2017.eng.155

14. Secretaría de Programación para la Prevención de la Drogadicción y la Lucha contra el Narcotráfico. Quinta Encuesta Nacional a Estudiantes de Enseñanza Media. 
Informe Final de Resultados [Internet]. 2012 [citado 14 de enero de 2021]. Disponible en: https://observatorio.gov.ar/media/k2/attachments/VZEst udioZNacionalZaZEstudiantesZdeZNivelZMedio.ZAoZ20121 . pdf

15. Última Hora. La ingesta excesiva de alcohol afecta más a las mujeres que a los hombres [Internet]. 2018 [citado 14 de enero de 2021]. Disponible en: https://www.ultimahora.com/la-ingesta-excesiva-alcoholafecta-mas-las-mujeres-que-los-hombres-n1128966.html

16. Fernandez Raone M. Adolescencia, consumo de sustancias y demanda terapéutica [Internet] [Tesis]. [Argentina]: Universidad Nacional de La Plata; 2017 [citado 14 de enero de 2021]. Disponible en: http://sedici.unlp.edu.ar/handle/10915/59898

17. Rueda-Jaimes GE, Pinto Ramírez JL, Rangel MartínezVillalba AM, Camacho López PA. Abuso de alcohol en niños y adolescentes escolarizados y factores asociados. Rev Colomb Psiquiatr. 2012;41(2):273-83. URL.

18. Fernández Díaz Y, Ortiz Martínez M, Aguilar Valdés J, Pérez Sosa D, Serra Larín S. Hábitos tóxicos en adolescentes y jóvenes con problemas de aprendizaje, 2011-2013. Rev Cuba Salud Pública. 2017;43(1):27-40. URL. 